\title{
BMJ Open Effect of polycystic ovary syndrome on cardiac autonomic function at a late fertile age: a prospective Northern Finland Birth Cohort 1966 study
}

\author{
Meri-Maija Ollila (D) , ${ }^{1}$ Antti Kiviniemi, ${ }^{2}$ Elisabet Stener-Victorin (D) , ${ }^{3}$ Mikko Tulppo, ${ }^{2}$ \\ Katri Puukka, ${ }^{4}$ Juha Tapanainen, ${ }^{1,5}$ Stephen Franks, ${ }^{6}$ Laure Morin-Papunen, ${ }^{1}$ \\ Terhi Piltonen ${ }^{1}$
}

To cite: Ollila M-M, Kiviniemi A, Stener-Victorin $\mathrm{E}$, et al. Effect of polycystic ovary syndrome on cardiac autonomic function at a late fertile age: a prospective Northern Finland Birth Cohort 1966 study. BMJ Open 2019;9:e033780. doi:10.1136/ bmjopen-2019-033780

- Prepublication history for this paper is available online. To view these files, please visit the journal online (http://dx.doi org/10.1136/bmjopen-2019033780).

Received 21 August 2019 Revised 11 November 2019 Accepted 12 November 2019

Check for updates

(c) Author(s) (or their employer(s)) 2019. Re-use permitted under CC BY-NC. No commercial re-use. See rights and permissions. Published by BMJ.

For numbered affiliations see end of article.

Correspondence to Dr Terhi Piltonen;

terhi.piltonen@oulu.fi

\section{ABSTRACT}

Objectives Previous studies of women in their 20 s and 30s have reported impaired autonomic function in women with polycystic ovary syndrome (PCOS). We aimed to study, for the first time, whether PCOS is associated with impaired cardiac autonomic function independent of metabolic and hormonal status in their late reproductive years.

Design A prospective Northern Finland Birth Cohort 1966 (NFBC1966) study including 5889 women born in 1966 and followed through the age of 46 . At that age, $n=3706 / 5123$ women $(72 \%)$ answered the postal questionnaires and $n=3280 / 5123$ women $(64 \%)$ participated in the clinical examination.

Setting General community.

Participants The sample included women presenting both irregular menses (oligomenorrhoea or amenorrhoea) and hirsutism at age $31(n=125)$ or with formally diagnosed PCOS by age $46(n=181)$ and women without PCOS symptoms or diagnosis $(n=1577)$.

Primary and secondary outcome measures Heart rate variability parameters: the root mean square of successive $\mathrm{R}-\mathrm{R}$ differences (rMSSD), spectral power densities (LF: low frequency and HF: high frequency) and baroreflex sensitivity (BRS).

Results We found that parasympathetic activity (assessed by rMSSD: 19.5 (12.4; 31.9) vs 24.3 (16.1; 34.8) $\mathrm{ms}, \mathrm{p}=0.004$ and HF: $172(75$; 399) vs 261 $\left.(112 ; 565) \mathrm{ms}^{2}, \mathrm{p}=0.002\right)$ and BRS $(6.13 \pm 3.12$ vs $6.99 \pm 3.52 \mathrm{~ms} / \mathrm{mm} \mathrm{Hg}, \mathrm{p}=0.036$ ) were lower in women with PCOS compared with the controls. However, in the multivariate regression analysis, PCOS, body mass index and the free androgen index did not significantly associate with rMSSD, whereas blood pressure, insulin resistance and triglycerides did.

Conclusions We report here for the first time that late reproductive-aged women with PCOS display impaired cardiac autonomic function manifested as decreased vagal activity. Metabolic status, rather than hyperandrogenaemia and PCOS per se, was the strongest contributing factor. Given the link between cardiac morbidity and impaired autonomic function, the findings underline the importance of screening and treating metabolic abnormalities early on in women with PCOS.

\section{Strengths and limitations of this study}

- This is the first study to investigate the cardiac autonomic function of late reproductive-aged women with polycystic ovary syndrome (PCOS).

- This study provides the largest study population by far compared with the previous studies in cardiac autonomic function in women with PCOS.

- We were able to adjust for many confounding factors and to study the effect of metabolic abnormalities.

The study is limited by the lack of PCOS phenotypes.

The study cannot be generalised with all ethnicities.

\section{INTRODUCTION}

Polycystic ovary syndrome (PCOS) is the most common endocrinopathy, affecting $6 \%-18 \%$ of women of reproductive age and characterised by irregular menstruation, clinical or biochemical hyperandrogenism and polycystic ovaries. ${ }^{1-3}$ Women with PCOS are commonly overweight or obese and typically present with insulin resistance, hyperinsulinaemia, increased blood pressure (BP), dyslipidaemia, metabolic syndrome and obstructive sleep apnoea, ${ }^{2}$ all of which are associated with impaired cardiac autonomic function. ${ }^{4-6}$ In the general population, the dysregulation of cardiac autonomic function has been associated with increased risk of many major global public health problems, such as depression, ${ }^{7}$ anxiety, ${ }^{8}$ hypertension, diabetes, cardiovascular diseases (CVDs) and mortality. ${ }^{9}$ Therefore, it is not surprising that women with PCOS have been shown to present with impaired cardiac autonomic function, that is, reduced parasympathetic (vagal) activity, ${ }^{10-12}$ and increased sympathetic nervous system activity. ${ }^{13} 14$ Previous researchers have used various methods, such as microneurography, the measurement of sympathetic skin responses, heart rate (HR) 
variability (HRV), HR recovery and a norepinephrine spill-over measurement, to assess the cardiac autonomic function in women with PCOS. Of these methods, the measurement of HRV (ie, variations in the time intervals between consecutive heartbeats) provides a wellestablished non-invasive method to assess, in particular, parasympathetic cardiac autonomic activity. ${ }^{15}$

During recent years, it has become an enigma whether women with PCOS have an increased risk for CVD. Taking into consideration that many PCOS traits, such as irregular cycles, hyperandrogenism and body mass index (BMI) difference from non-PCOS controls, seem to diminish with age,${ }^{16}$ it is also important to assess CVD-related traits, such as cardiac autonomic function, in women with PCOS in the late reproductive years and beyond menopause to elucidate their possible risk for CVD outcomes. Previous studies on cardiac autonomic function have included women with PCOS in their $20 \mathrm{~s}$ or $30 \mathrm{~s}$, but to date, no studies have been carried out on women in their late reproductive years. Moreover, the populations studied have been derived from PCOS clinics; thus, communitybased studies are needed. Therefore, the main aim of this study was to investigate whether women with PCOS from a general population display reduced HRV as an indicator of impaired cardiac autonomic function during their late reproductive years (at age 46). Additionally, we investigated the role of confounding metabolic abnormalities, such as excess weight, abdominal obesity, hyperandrogenism, increased BP, dyslipidaemia and insulin resistance, in the cardiac autonomic function in affected women.

\section{MATERIALS AND METHODS \\ Study population}

The study population comprises the Northern Finland Birth Cohort 1966 (NFBC1966), which is a large, prospective, general population-based, longitudinal birth cohort. All individuals with expected births during 1966 in the two northernmost provinces in Finland (Oulu and Lapland) were included in this birth cohort (12231 births, 5889 women, $96.3 \%$ of all births during 1966 in that area). Enrolment in this database began at the 24th gestational week, and the women were followed through age 46. The follow-up protocol of the cohort was previously described in detail, and the main data collection points during adulthood were carried out at ages 31 and $46 .{ }^{17}$ Briefly, postal questionnaires were sent to all living cohort members with known addresses at ages 31 (81\% answered, $\mathrm{n}=4523 / 5608)$ and $46(72 \%$ answered, $\mathrm{n}=3706 / 5123)$ to collect information about health, behaviour and social background. Postal questionnaires included an invitation to participate in clinical examinations at ages 31 (77\% participation rate, $\mathrm{n}=3127 / 4074)$ and 46 (64\% participation rate, $\mathrm{n}=3280 / 5123)$. Weight and height were self-reported at age 14 (with the help of the participants' parents) and clinically measured at ages 31 and 46 . BMI was calculated as the ratio of weight $(\mathrm{kg})$ and height squared $\left(\mathrm{m}^{2}\right)$. Besides anthropometric measurements, the clinical examinations at age 46 included blood sampling and assessments of cardiovascular health status, including systolic and diastolic BP (SBP and DBP, respectively), carotid and cardiac ultrasound and evaluations of HRV and baroreflex sensitivity (BRS). Waist circumference was measured at the level midway between the lowest rib margin and the iliac crest. Brachial SBP and DBP were measured three times with $1 \mathrm{~min}$ interval after $15 \mathrm{~min}$ rest by an automated, oscillometric BP device with an appropriately sized cuff (Omron Digital Automatic Blood Pressure Monitor Model M10-IT; Omron, Kyoto, Japan), and SBP and DBP averages were calculated. ${ }^{18}$ The level of glucose metabolism was classified according to WHO standards, ${ }^{19}$ based on a 2-hour oral glucose tolerance test (performed at age 46) and a previously established diagnosis of type 2 diabetes. ${ }^{20}$

\section{Definition of PCOS and control groups}

At age 31, PCOS symptoms (ie, oligomenorrhoea/amenorrhoea and hirsutism) were self-reported. Of all the women who responded to questions regarding PCOS symptoms $(\mathrm{n}=4523)$, after excluding pregnant women and those using hormonal preparations $(\mathrm{n}=1459)$ or not permitting the use of their data $(n=41), 4.1 \% \quad(n=125)$ reported both oligomenorrhoea/amenorrheoa and hirsutism. The validity of this questionnaire to distinguish PCOS cases with typical hormonal, metabolic and psychological traits characteristic to the syndrome, as well as ovarian morphology for PCOS, has previously been described. ${ }^{20-23}$ At age 46 , the postal questionnaire included a question on existing PCOS diagnosis, to which 181 subjects responded 'yes'. Consequently, the women reporting both oligomenorrhoea/amenorrhoea and hirsutism and/or reporting PCOS diagnosis by age 46 were considered cases $(n=279)$. Women without PCOS symptoms at age 31 and without diagnosis of PCOS by age 46 were considered controls $(n=1577)$. The characteristics of the PCOS and control populations and the flowchart of the formation of the PCOS and control groups have previously been described. ${ }^{17}$

\section{Evaluation of cardiac autonomic function}

A flowchart of the study is presented in figure 1. HRV was measured in the study subjects at age 46 in the research unit at Oulu University Hospital and in two other major hospitals nearby. The subjects were informed about the measurement protocol, and an HR monitor (RS800CX, Polar Electro Oy, Kempele, Finland) to record R-R intervals (RRi) and a standard lead-II ECG (Cardiolife, Nihon Kohden, Tokyo, Japan) were placed on the subjects while seated. Also, breathing frequency (MLT415/D, Nasal Temperature Probe, ADInstruments, Bella Vista, New South Wales, Australia) and BP by finger photoplethysmography (Nexfin, BMEYE Medical Systems, Amsterdam, the Netherlands) were recorded with a sampling frequency of $1000 \mathrm{~Hz}$ (PowerLab 8/35, ADInstruments). These preparations were followed by at 


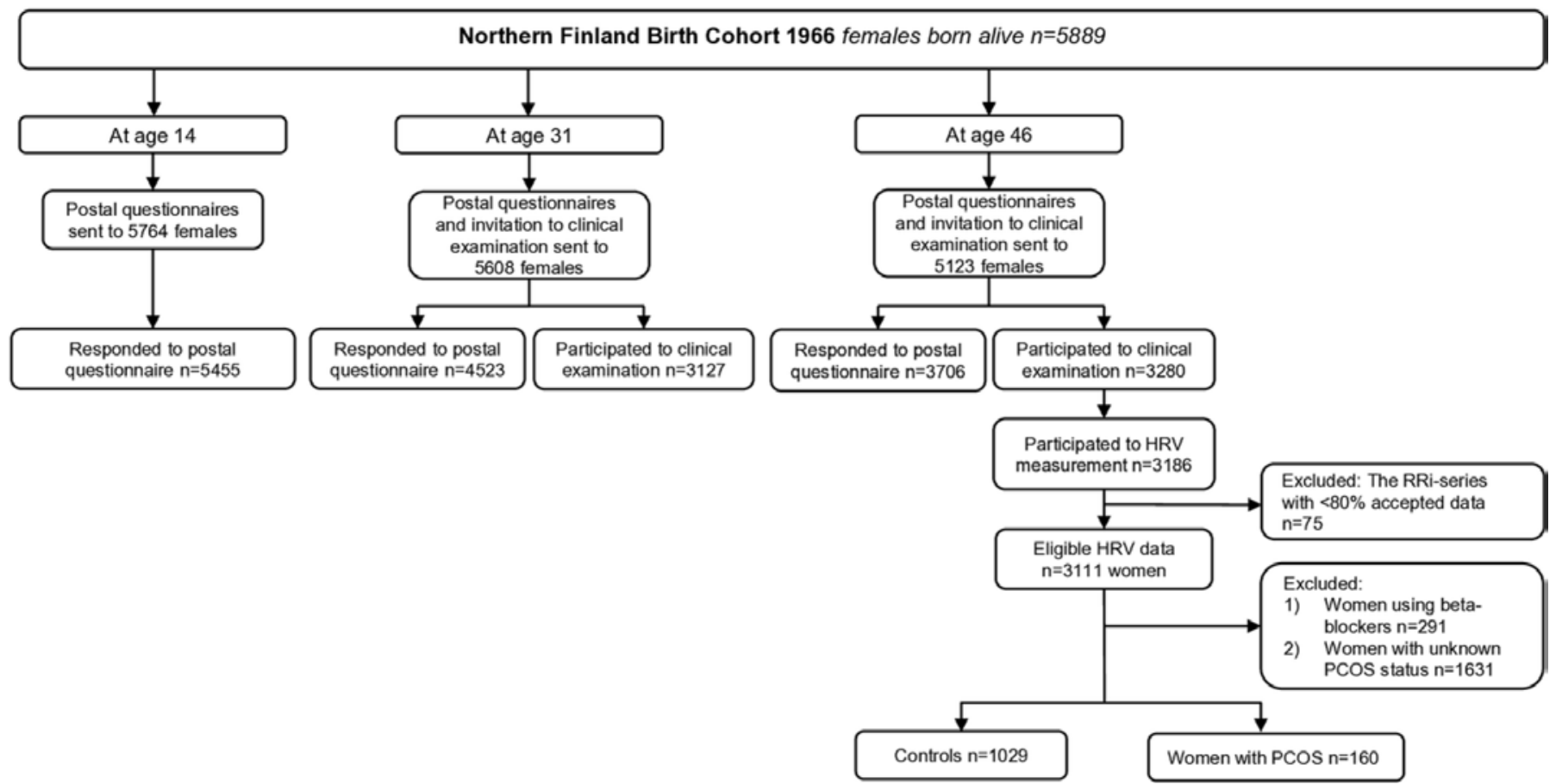

Figure 1 Flowchart of the study. HRV, heart rate variability; PCOS, polycystic ovary syndrome; RRi, R-R interval.

least 1 min stabilisation period before the beginning of the 3 min recording period in the seated position. After that recording period, the participants stood up and remained still in a standing position for another $3 \mathrm{~min}$ while breathing normally.

\section{Analysis of HRV}

The first $150 \mathrm{~s}$ of recording in a seated position and the last $150 \mathrm{~s}$ in a standing position were used in the analyses. The RRi data were edited based on visual inspections, and the artefacts and ectopic beats were removed and replaced according to the local average (Hearts 1.2, University of Oulu, Oulu, Finland). Sequences with $\geq 10$ consecutive beats of noise or ectopic beats were deleted. The RRi series with $\geq 80 \%$ accepted data were included in the analyses. The final study population included 1029 controls and 160 women with PCOS. Mean HR, rMSSD (the root mean square of successive R-R differences) and spectral power densities (fast Fourier transformation, length 512 beats), including low frequency (LF: $0.04-0.15 \mathrm{~Hz}, \mathrm{~ms}^{2}$ ) and high frequency (HF: $0.15-0.40 \mathrm{~Hz}, \mathrm{~ms}^{2}$ ) components of HRV and their ratio (LF/HF), were analysed. While rMSSD and HF component of HRV are mainly determined by cardiac parasympathetic activity, LF component of HRV is affected largely by parasympathetic activity but also includes effects of sympathetic activity as well as other unidentified factors. ${ }^{152}$ The LF/HF ratio has been used as a marker of sympatho-vagal balance, particularly during orthostatic stimulus. ${ }^{25}$ However, as the physiological background of LF component of HRV is complex, the conclusions concerning sympatho-vagal balance by LF/ HF are limited. ${ }^{24}$

\section{Analysis of BRS}

BRS was assessed in the participants who had the measures performed at the Oulu University Hospital (609 controls and 105 women with PCOS). Continuous ECG, BP and respiration signals were imported to custom-made, stand-alone Matlab-based software (Biosignal Processing Team, University of Oulu, Oulu, Finland), with which $\mathrm{RRi}$ and SBP values were extracted. Artefacts and ectopic beats were replaced using linear interpolation $(<5 \%$ for accepted recording) and, thereafter, resampled at $2 \mathrm{~Hz}$ and detrended $(<0.04 \mathrm{~Hz}$, Savitzky-Golay method $)$. A fast Fourier transform (Welch method, segments of 128 samples with $50 \%$ overlap) was performed to analyse the LF $(0.04-0.15 \mathrm{~Hz})$ power of RRi and SBP oscillations for subsequent analysis of BRS using the alpha method if sufficient coherence ( $\geq 0.5$ ) between LF oscillations in RRi and SBP was verified. The present BRS method quantifies cardiac autonomic responses to spontaneous SBP variation, detected by baroreceptors in the aortic arch and the carotid sinus, which include both parasympathetic and sympathetic effects. ${ }^{26}$ Concurrently, the LF oscillation of $\mathrm{BP}\left(\mathrm{LF}_{\mathrm{SBP}}, 0.04-0.15 \mathrm{~Hz}\right)$ was obtained and considered as a surrogate for peripheral sympathetic activity. However, the physiological background of $\mathrm{LF}_{\mathrm{SBP}}$ is not fully established, as there are competing theories of central oscillation of sympathetic drive and BRS resonance. ${ }^{27}$

\section{Laboratory methods}

The laboratory methods have previously been described in detail. ${ }^{17}$ At age 46, sex hormone binding globulin (SHBG) was assayed by chemiluminometric immunoassay (Immulite 2000, Siemens Healthcare, Llanberis, UK). The serum samples for testosterone $(\mathrm{T})$ were assayed 
using Agilent triple quadrupole 6410 LC/MS equipment (Agilent Technologies, Wilmington, Delaware, USA). The free androgen index (FAI) was calculated using the following equation: $(100 \times \mathrm{T}) / \mathrm{SHBG}$. The serum total cholesterol, the high-density lipoprotein (HDL), the lowdensity lipoprotein (LDL) and triglycerides were determined using an enzymatic assay method. Fasting plasma glucose (f-gluc) was analysed by an enzymatic dehydrogenase method (methods of cholesterol, HDL, LDL, triglycerides and f-gluc: Advia 1800, Siemens Healthcare Diagnostics, Tarrytown, New York, USA). Fasting serum insulin (f-ins) was analysed by a chemiluminometric immunoassay (Advia Centaur XP, Siemens Healthcare Diagnostics, Tarrytown, New York, USA). The f-gluc and f-ins values were used to calculate the Homeostasis Model Assessment-insulin resistance (HOMA-IR) index (f-gluc $\times$ f-ins/22.5). The high-sensitivity C-reactive protein (hsCRP) was analysed by an immune-nephelometric assay (BN ProSpec, Siemens Healthcare Diagnostics, Newark, Delaware, USA). The samples were analysed at NordLab Oulu, a testing laboratory (T113) accredited by the Finnish Accreditation Service (FINAS) (EN ISO 15189).

\section{Hopkins Symptom Check List-25}

Hopkins Symptom Check List-25 Part 1 includes 10 items that check for anxiety symptoms, and this part was used in the present study. ${ }^{28}$

\section{Statistical methods}

Women using beta-blockers (104 controls $(7.7 \%)$ and 30 women with PCOS (13.3\%), $\mathrm{p}=0.009)$ were excluded from the HRV analysis. Continuous data were presented as mean with SD or as median with $25 \%$ and $75 \%$ quartiles. Continuous variables with skewed distributions were transformed into a natural logarithm (ln). Differences in normally distributed continuous parameters were analysed using the Student's t-test, whereas the Mann-Whitney U-test was used in the case of skewed distribution. Categorical data were reported as prevalence with the number of cases, and the difference between the study groups was analysed by cross tabulation and the $\chi^{2}$ test or the Fisher's exact test, when appropriate. The mean arterial pressure (MAP) was calculated as follows: $\mathrm{DBP}+\frac{1}{3}(\mathrm{SBP}-\mathrm{DBP})$.

Univariate and multivariate linear regression analyses were used to study the factors associated with the HRV parameters. First, univariate linear regression models were used to reveal the parameters that were significantly associated with the outcome variable. Then, stepwise multivariate models were used to identify the most important explanatory variables. The final multivariate model included the following variables as explanatory variables: PCOS, BMI at age 46, MAP, FAI, HOMA-IR and triglycerides. The number of explanatory variables included in the final model had to be limited to avoid multicollinearity. BMI was included in the model, as it significantly differs between the PCOS and control women, and obesity is suggested to affect HRV. ${ }^{4}$ MAP was selected because it combines information from both
SBP and DBP; FAI was included in the model because it is considered a good indicator of hyperandrogenaemia in women with $\mathrm{PCOS}^{2}$ and hyperandrogenaemia has been suggested to alter HRV in women with PCOS. ${ }^{14}$ The homeostasis model assessment for insulin resistance was used as an estimate of insulin resistance, as it combines information from both fasting insulin and glucose levels, and triglycerides was included, as hypertriglyceridaemia is a typical lipid abnormality in PCOS women and is linked to CVD risks. ${ }^{29}$ Anxiety was not included in the final multivariate model, because in the preliminary models using the stepwise method it was always the first variable to be excluded. The results of linear regression models are reported as unstandardised coefficients (B), $95 \%$ confidence intervals for $B, p$ values and $R^{2}$ values for the model. The multicollinearity assumptions of the multivariate linear regression model were investigated using variance inflation factor (VIF), tolerance and eigenvalue indexes. In addition, a histogram of regression standardised residual frequency, normal P-P plot of regression-standardised residuals and scatter plot figures were visually inspected to ensure that the model met the assumptions in the analysis. The data were analysed using SPSS software (IBM SPSS Statistics V.24.0, IBM Corp.). A $p$ value $<0.05$ was considered statistically significant.

All the participants took part on a voluntary basis and signed informed consent forms.

\section{Patient and public involvement statement}

The patients, the public or any third parties were not involved in the design, conduct, reporting or dissemination of our research.

\section{RESULTS}

\section{HRV and BRS}

When compared with the control women, the women with PCOS in a seated position had a significantly higher mean HR and significantly lower values of $\mathrm{rMSSD}, \mathrm{LF}_{\mathrm{RRi}}$, $\mathrm{HF}_{\mathrm{RRi}}$ and BRS (figure 2). However, when adjusting for BMI at age 46, the women with PCOS had lower values only in rMSSD $(\mathrm{p}=0.033)$ and $\mathrm{HF}_{\mathrm{RRi}}(\mathrm{p}=0.016)$ compared with the controls. In the standing position, the women with PCOS did not differ from the controls after adjustment for BMI at age 46 (data not shown).

\section{HRV according to BMI group}

After dividing the women according to BMI ( $<25$ or $\geq 25 \mathrm{~kg}$ / $\mathrm{m}^{2}$ ), the lean women with PCOS did not differ from the lean control women regarding the HRV parameters, whereas the overweight/obese women with PCOS had higher HRs and lower rMSSD, $\mathrm{LF}_{\mathrm{RRi}}$ and $\mathrm{HF}_{\mathrm{RRi}}$ compared with the overweight/obese controls (figure 3). It is noteworthy that the overweight/obese women with PCOS had higher BMIs and waist circumferences and had abnormal glucose metabolism and hyperandrogenaemia more often than the overweight/obese control women (data not shown). 
A. HR

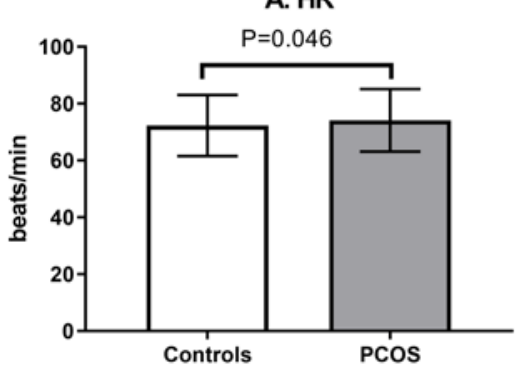

C. $\mathrm{LF}_{\mathrm{RRi}}$

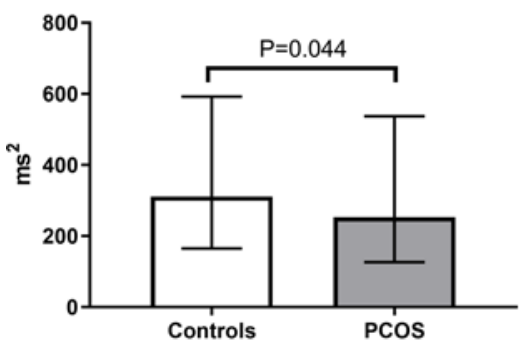

E. LF/HF RRi $^{- \text {ratio }}$

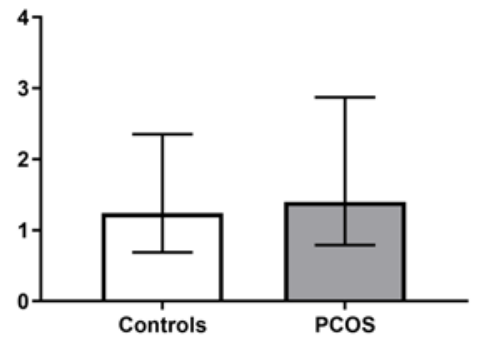

G. LF $_{\text {SBP }}$

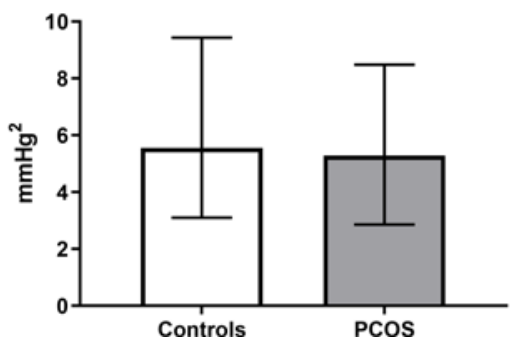

B. RMSSD
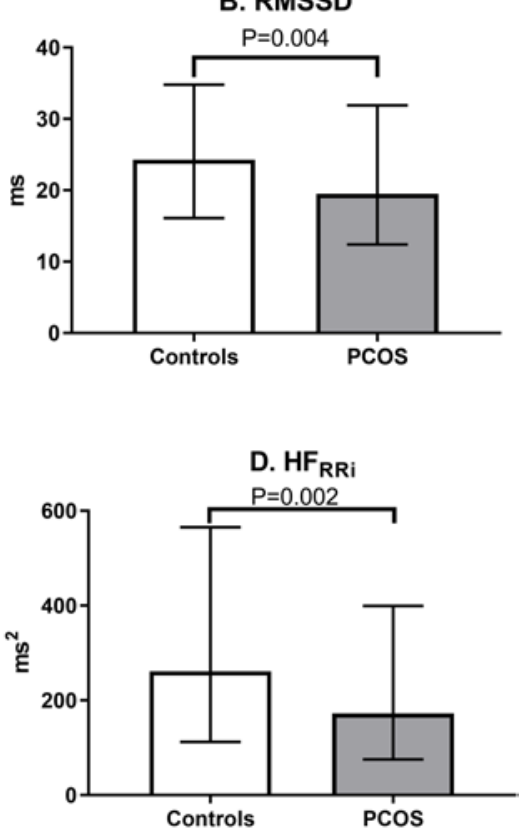

F. $\alpha_{1}$

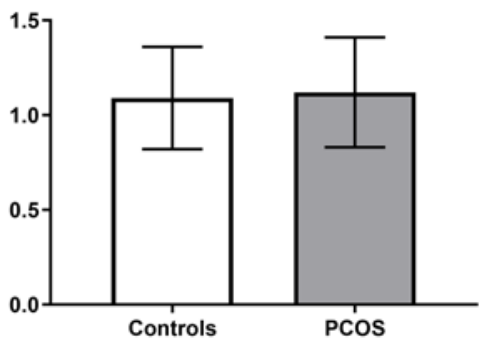

H. BRS

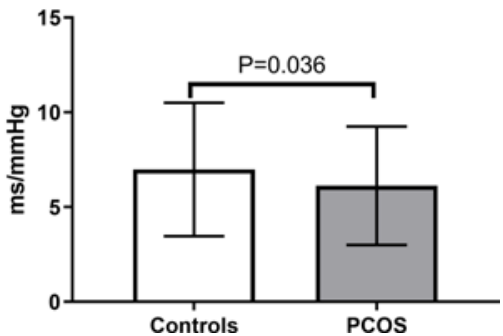

Figure 2 Heart rate variability parameters in controls and in women with PCOS at age 46 in seated position. The users of betablockers were excluded. Values are mean \pm SD or median with $25 \%$ and $75 \%$ quartiles, and the significance testing was made by Student's t test (In-transform was made to achieve normality). HR, heart rate; rMSSD, the root mean square of successive R-R differences; $L_{\text {RRi }}$, low-frequency $(0.04-0.15 \mathrm{~Hz})$ power; $\mathrm{HF}_{\mathrm{RRi}}$, high-frequency $(0.15-0.4 \mathrm{~Hz})$ power; $\alpha_{1}$, short-term fractal-like scaling exponent by detrended fluctuation analysis; SBP, systolic blood pressure; BRS, baroreflex sensitivity; PCOS, polycystic ovary syndrome.

We also further divided the population into overweight (BMI $25-30 \mathrm{~kg} / \mathrm{m}^{2}$ ) and obese $\left(\mathrm{BMI}>30 \mathrm{~kg} / \mathrm{m}^{2}\right)$ groups. We found that the overweight women with PCOS $(n=55)$ had significantly lower rMSSD $(2.54 \pm 0.6$ vs $2.90 \pm 0.6$, $\mathrm{p}=0.006), \mathrm{LF}_{\text {RRi }}(4.91 \pm 0.9$ vs $5.52 \pm 0.9, \mathrm{p}=0.034)$ and $\mathrm{HF}_{\text {RRi }}$ $(4.30 \pm 1.2$ vs $5.00 \pm 1.2, \mathrm{p}=0.010)$ compared with the overweight control women $(\mathrm{n}=328)$, whereas HR $(80.0 \pm 11.2$ vs $75.8 \pm 9.6, \mathrm{p}=0.064), \mathrm{LF} / \mathrm{HF}$ ratio $(0.61 \pm 0.9$ vs $0.51 \pm 0.9$, $\mathrm{p}=0.187), \mathrm{LF}_{\mathrm{SBP}}(1.51 \pm 0.8$ vs $1.80 \pm 0.8, \mathrm{p}=0.087)$, BRS $(1.67 \pm 0.4$ vs $1.81 \pm 0.5, \mathrm{p}=0.182)$ and $\alpha(1.68 \pm 0.4$ vs $1.81 \pm 0.5, \mathrm{p}=0.182)$ did not differ between these two groups. We found no significant differences between the obese PCOS and control women in any HRV parameters, but this might be due to a lack of statistical power, as our sample included only 23 obese women with PCOS (data not shown). 
A. HR

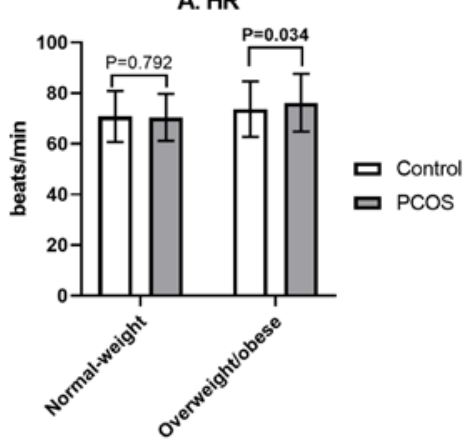

C. $\mathrm{LF}_{\mathrm{RRi}}$

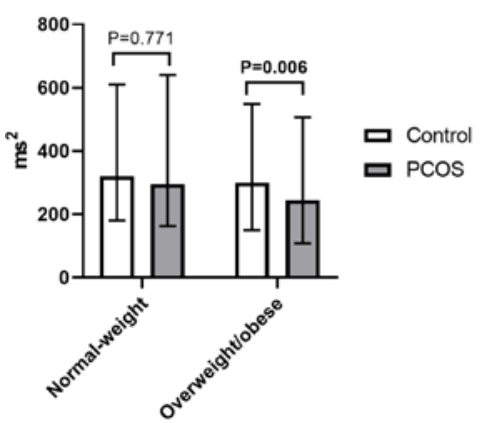

E. LF/HF-ratio

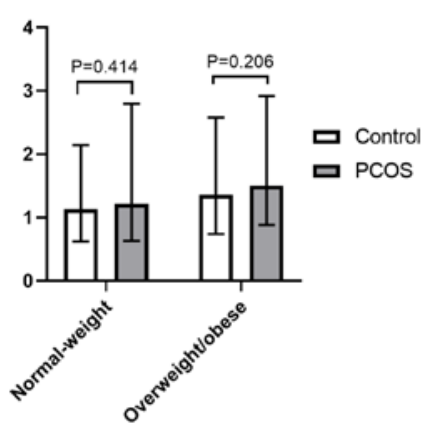

G. $\mathrm{LF}_{\mathrm{SBP}}$

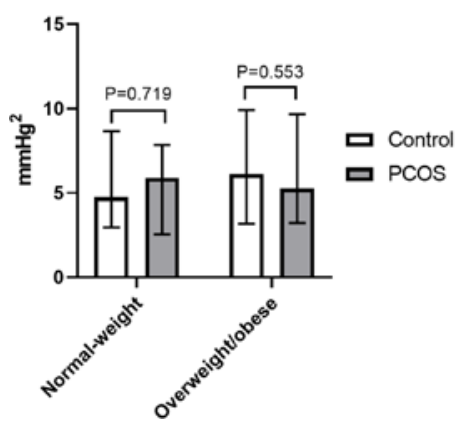

B. RMSSD

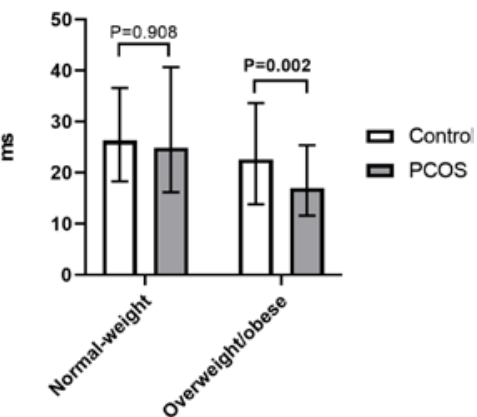

D. $\mathrm{HF}_{\mathrm{RRi}}$

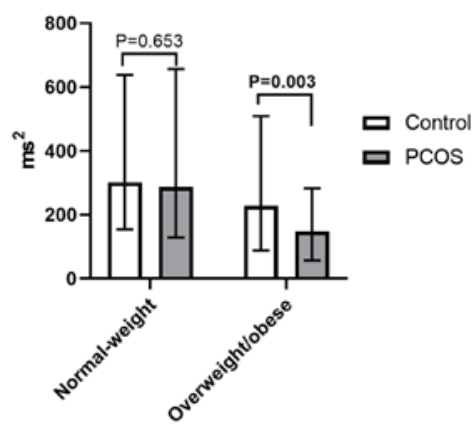

F. $\alpha_{1}$

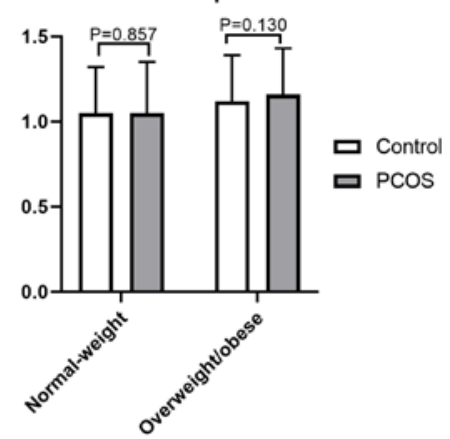

H. BRS

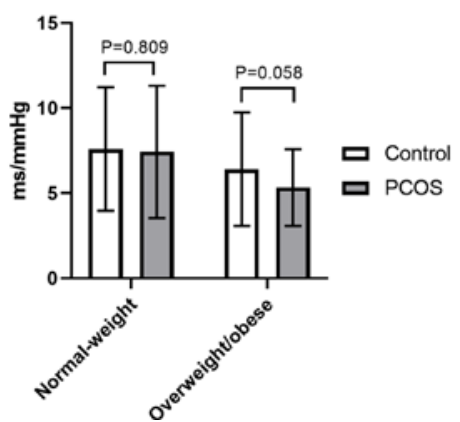

Figure 3 Heart rate variability parameters in controls and in women with PCOS at age 46 according to the BMI group. The assessment of autonomic function in seated position. The users of beta blockers were excluded. Values are mean \pm SD or median with $25 \%$ and $75 \%$ quartiles, and the significance testing was made by Student's t-test (In-transform was made to achieve normality). Statistically significant $p$ values are bolded. HR, heart rate; rMSSD, the root mean square of successive R-R differences; $\mathrm{LF}_{\mathrm{RB}}$; low-frequency $(0.04-0.15 \mathrm{~Hz})$ power; $\mathrm{HF}_{\mathrm{BR}}$, high-frequency $(0.15-0.4 \mathrm{~Hz})$ power; $\alpha_{1}$, short-term fractal-like scaling exponent by detrended fluctuation analysis; SBP, systolic blood pressure; BRS, baroreflex sensitivity; PCOS, polycystic ovary syndrome. 


\section{Linear regression analysis for rMSSD}

The univariate linear regression analysis demonstrated that rMSSD was associated with BMI at ages 31 and 46 and with waist circumference, anxiety, SBP, DBP, MAP and the serum levels of total cholesterol, HDL, LDL, triglycerides, glucose, insulin, HOMA-IR, SHBG, FAI and hsCRP at age 46 (table 1 ). The BMI at age 14 was not associated with rMSSD. The multivariate linear regression analysis demonstrated that MAP, HOMA-IR and triglycerides were the strongest explanatory variables for rMSSD (table 1).

\section{Hyperandrogenaemia}

Total or calculated free $\mathrm{T}$ at age $46 \mathrm{did}$ not associate with rMSSD in the linear regression analysis (data not shown). The FAI was negatively associated with rMSSD ( $\mathrm{B}=-0.121,95 \%$ CI: -0.199 to $-0.042, \mathrm{p}=0.003)$, but lost its significance after an adjustment for BMI. Similarly, the serum level of SHBG was positively associated with $\mathrm{rMSSD}$ ( $\mathrm{B}=0.001,95 \%$ CI: 0.001 to $0.002, \mathrm{p}<0.001)$, but lost its significance after a BMI adjustment.

\section{DISCUSSION}

To our knowledge, this is the first study to investigate the cardiac autonomic function of late reproductive age women with PCOS. We demonstrate here, in a large general population-based setup, that late reproductive aged women with PCOS display reduced HRV, indicating reduced parasympathetic activity. However, in the multivariate linear regression analysis, the reduced HRV was associated with elevated $\mathrm{BP}$, insulin resistance and dyslipidaemia, but not PCOS per se, demonstrating that metabolic abnormalities are likely to be the main cause for reduced HRV in women with PCOS.

Previous studies have reported that women with PCOS display impaired cardiac autonomic function with decreased parasympathetic and increased sympathetic activity. ${ }^{10-12}$ In line with our findings, a study of 75 overweight women with PCOS and 75 age and BMI-matched controls suggested that impaired HR recovery, a marker for decreased parasympathetic activity, was caused by excess weight and insulin resistance and not by PCOS per se ${ }^{30}$ Furthermore, a cross-sectional study of 31 PCOS

Table 1 Univariate and multivariate linear regression models for heart rate variability measure (rMSSD) in women with PCOS

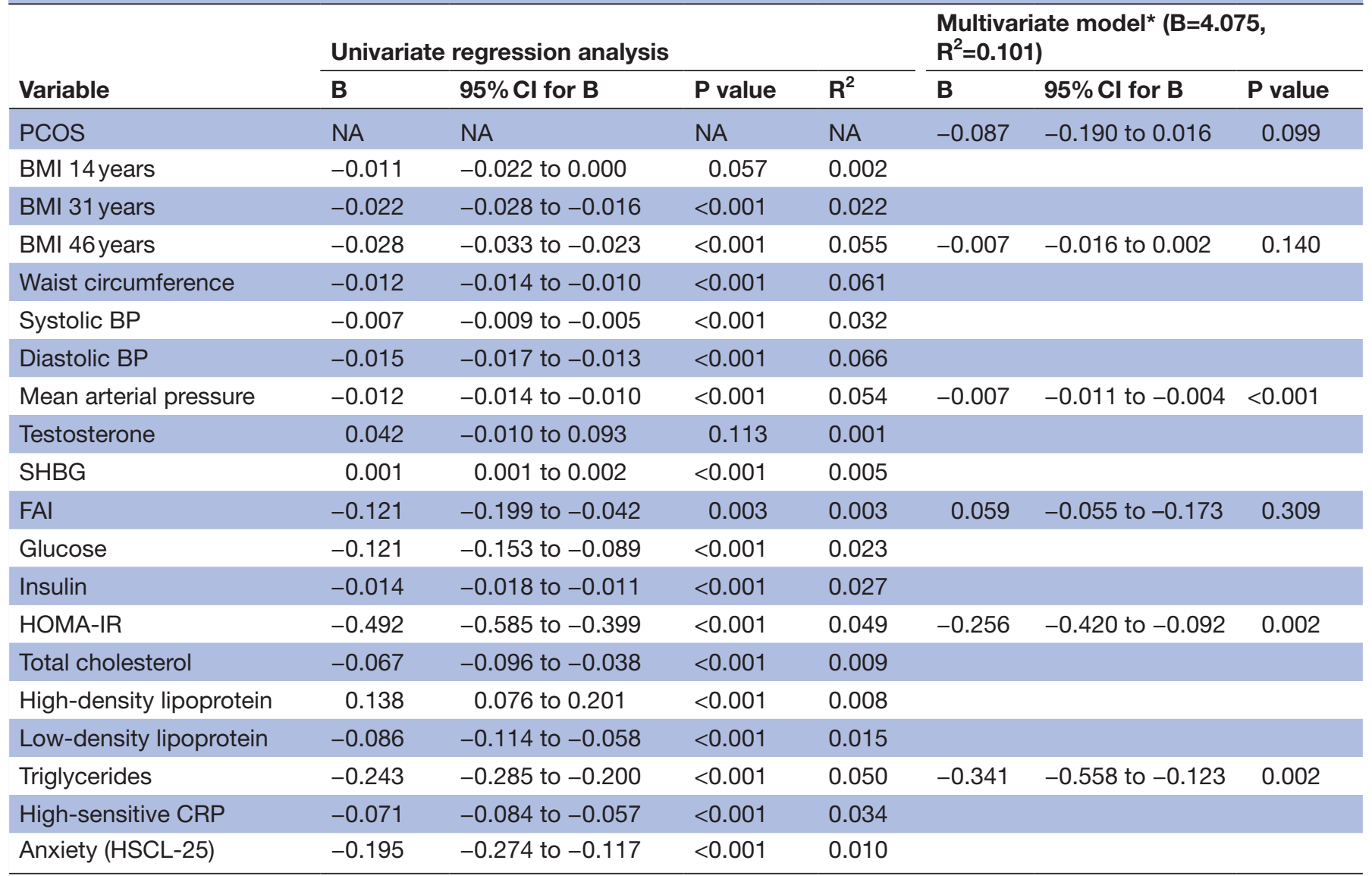

*The multivariate model included PCOS, BMI, mean arterial pressure, FAI, HOMA-IR and triglycerides as explanatory variables. B, unstandardized coefficient from linear regression analysis.

BMI, body mass index; BP, blood pressure; CRP, C-reactive protein; FAI, free androgen index; HOMA-IR, homeostasis model assessmentinsulin resistance; NA, not applicable; PCOS, polycystic ovary syndrome; rMSSD, the square root of the mean squared differences of successive normal-to-normal RR intervals (RRi); SHBG, sex hormone binding globulin. 
cases recruited from outpatient clinics reported that women with PCOS showed significantly decreased vagal activity, but the role of confounding metabolic abnormalities was not assessed, even though the women with PCOS had significantly higher BMIs, waist-hip ratios, BP and serum levels of $\mathrm{T}$ and glucose than the control women. ${ }^{10}$ Our findings are also in line with previous studies conducted in the general population in which an association between BP, glucose metabolism, dyslipidaemia and cardiac autonomic function was reported. ${ }^{531}$

In the present study, the overweight/obese women with PCOS had more adverse changes in HRV parameters than the overweight/obese control women, whereas the lean PCOS and control groups had comparable HRV values. However, the difference between the overweight/obese PCOS and control groups may have reflected the higher mean BMIs and waist circumferences and the higher prevalence of abnormal glucose metabolism in the overweight/obese PCOS group than in the overweight/obese controls. Previous studies have reported conflicting results regarding the effect of BMI on autonomic function in PCOS. A study of 19 overweight/obese women with PCOS and 21 overweight/obese control women reported that the women with PCOS had elevated muscle sympathetic nervous activity (MSNA), whereas HRV parameters did not significantly differ between the groups. ${ }^{13}$ However, it was reported that the non-obese $\left(\mathrm{BMI}<30 \mathrm{~kg} / \mathrm{m}^{2}\right.$ ) control and PCOS groups did not have significantly different SD of all RRis (SDNN), rMSSD or percentage of successive differences in RRi $>50 \mathrm{~ms}$ (pNN50), whereas the obese (BMI $\geq 30 \mathrm{~kg} / \mathrm{m}^{2}$ ) women with PCOS had significantly decreased SDNN and pNN50, but not rMSSD. ${ }^{32}$ The findings of that study also demonstrated that sympathetic skin responses, investigated by electromyography from the median or tibial nerve, were altered in both the non-obese and obese PCOS groups. These conflicting results might be explained by the fact that the effect of obesity in sympathetic activation might be regional, as obesity has been reported to increase sympathetic activity in the kidneys and skeletal muscle vasculature but to reduce it in the heart. ${ }^{33}$

We found that serum T or FAI at age 46 did not associate with HRV after BMI adjustment (nor did FAI or $\mathrm{T}$ at age 31, data not shown). In line with our findings, two previous studies found no significant association between $\mathrm{T}$ and $\mathrm{LF}^{12}$ or hormonal profile and $\mathrm{HR}$ recovery. ${ }^{11}$ By contrast, the total $\mathrm{T}$ level was reported to be inversely associated with $\mathrm{LF}$ and LF/HF ratio in the women with PCOS during mental stress testing. ${ }^{34}$ However, in the present study, we used the golden standard, a liquid-tandem mass-spectrometry assay for $\mathrm{T}$ measurement, whereas the previous studies have used immunoassays. ${ }^{1134}$ Another study found higher MSNA in the normal weight women with PCOS compared with the normal weight controls, and the strongest explanatory factors for higher MSNA in the women with PCOS were total and free T and cholesterol. ${ }^{14}$ However, MSNA describes sympathetic activity, whereas HRV mainly describes parasympathetic activity.
Also, the phenotype of PCOS was suggested to influence cardiac autonomic function, as the anovulatory women with PCOS showed lower HRV response in mental stress tests than the controls, whereas the ovulatory PCOS women showed intermediate values. ${ }^{34}$ However, in that study, the women with anovulatory PCOS had a different metabolic profile than those with ovulatory PCOS and the control groups. ${ }^{34}$ Furthermore, previous studies have indicated that anxiety was associated with reduced HRV in the general population. ${ }^{8}$ In our analysis, anxiety had a weak association with rMSSD in the univariate linear regression model, although a further analysis revealed that metabolic abnormalities played a more important role in the reduction of vagal activity.

A recent study addressed the inter-related effects of insulin resistance, hyperandrogenism, chronic inflammation and sympathetic dysfunction (evaluated by MSNA) in 49 PCOS and 23 control women; based on the findings, the authors concluded that sympathetic dysfunction and hyperandrogenism were associated with PCOS and that chronic inflammation might be the mediating factor between sympathetic function, hyperandrogenism and insulin resistance. ${ }^{35}$ However, in the present study, the surrogate marker of sympathetic activity, $\mathrm{LF}_{\mathrm{SBP}}$, did not significantly differ between the PCOS and control women, suggesting that in our population the women with PCOS would not have increased sympathetic activity. This needs to be interpreted with caution as $\mathrm{LF}_{\mathrm{SBP}}$ does not directly measure sympathetic activity. The frequency width of $0.04-0.15 \mathrm{~Hz}$ used in the present study could be considered a limitation, as a frequency width of $0.075-0.15 \mathrm{~Hz}$ is affected by sympathetic modulation. ${ }^{36}$ However, the LF oscillation of BP usually has a central frequency at $\sim 0.1 \mathrm{~Hz}$ which considerably varies in relation to sympathetic effect, ${ }^{3738}$ supporting the use of a wider spectral band for $\mathrm{LF}_{\mathrm{SBP}}$. Of note, in our data, the women with PCOS had significantly lower BRS, but the significance disappeared after adjusting for BMI, indicating that PCOS per se does not affect BRS.

The strength of our study is that it includes by far the largest sample size of women with PCOS and HRV measurements. The data also add to the literature by representing a community-based approach. Moreover, we were able to adjust for many confounding factors and to study the effect of metabolic abnormalities. Also, this is the first study to investigate women with PCOS at a late fertile age. The definition of the PCOS population could be considered a limitation; however, we have previously shown that the population does display the typical endocrine, metabolic and psychological profiles of PCOS. ${ }^{21-23}$ Moreover, a recent genome-wide metaanalysis reported that the genetic architecture does not differ based on the diagnostic criteria used for PCOS (self-reported, NIH criteria or non-NIH Rotterdam criteria), ${ }^{39}$ thus supporting our approach. Our study population included only women with Caucasian ethnicity; consequently, our results are best generalised to PCOS women with Caucasian ethnicity, as ethnicity 
is known to affect many traits of PCOS. In addition, a longer HRV recording period would have been more favourable, but due to logistical reasons and the large number of subjects to measure we had to limit the time. However, the recording period of $150 \mathrm{~s}$ used in the present study should be reliable. ${ }^{15}$

In conclusion, in this community-based data set, the women with PCOS displayed reduced vagal activity at a late fertile age with a strong association with metabolic abnormalities. The fact is that impaired cardiovascular autonomic function (ie, increased sympathetic/ decreased parasympathetic activity) reflects the risk for cardiac morbidity; also this is most likely the case in PCOS. This underlines the importance of the active screening and treatment of metabolic abnormalities in women with PCOS, as also suggested by the recently published guidelines for PCOS. ${ }^{40}$ Previous studies have reported that in overweight/obese women with PCOS, the impaired cardiovascular autonomic function could be improved by a 10 -week energy restriction, ${ }^{41}$ a 3 -month aerobic exercise training programme ${ }^{42}$ or acupuncture. ${ }^{43}$ Importantly, it has been shown that the autonomic disturbance can be reversed with weight reduction, ${ }^{44}$ which should justify increasing resources and efforts targeting weight management.

\section{Author affiliations}

${ }^{1}$ Department of Obstetrics and Gynaecology, University of Oulu and Oulu University Hospital, Medical Research Centre, PEDEGO Research Unit, Oulu, Finland ${ }^{2}$ Research Unit of Internal Medicine, Medical Research Centre Oulu, University of Oulu and Oulu University Hospital, Oulu, Finland

${ }^{3}$ Department of Physiology and Pharmacology, Karolinska Institutet, Stockholm, Sweden

${ }^{4}$ NordLab Oulu, Department of Clinical Chemistry, University of Oulu and Oulu University Hospital, Medical Research Centre Oulu, Oulu, Finland

${ }^{5}$ Department of Obstetrics and Gynaecology, University of Helsinki and Helsinki University Hospital, Helsinki, Finland

${ }^{6}$ Institute of Reproductive and Developmental Biology, Imperial College London, London, UK

Acknowledgements We thank the late Professor Paula Rantakallio for establishing the NFBC, the participants in the 31 and 46-year studies and the NFBC Project Centre.

Contributors MMO: study design, data analyses and interpretation, manuscript drafting and revision; AK: study design, data collection, analyses and interpretation, manuscript drafting and revision; ESV and LMP: data interpretation, manuscript drafting and revision; MT: data collection and interpretation, manuscript revision; KP: laboratory analyses and manuscript revision; JST and SF: data interpretation and manuscript revision and TTP: study design, data interpretation, manuscript drafting and revision.

Funding This work was supported by grants from the Finnish Medical Foundation, the North Ostrobothnia Regional Fund, the Academy of Finland (project grants $315921,321763,104781,120315,129269,1114194,24300796)$, the Center of Excellence in Complex Disease Genetics, SALVE, the Sigrid Jusélius Foundation, Biocenter Oulu, University Hospital Oulu and the University of Oulu (75617), Medical Research Center Oulu, the National Institute for Health Research (UK), NHLBI grant 5R01HL087679-02 through the STAMPEED program (1RL1MH08326801), NIH/NIMH (5R01MH63706:02), the ENGAGE project and grant agreement HEALTH-F4-2007-201413, EU FP7 EurHEALTHAgeing (277849), the Medical Research Council UK (G0500539, G0600705, G1002319, G0802782, PrevMetSyn/ SALVE), the MRC, the Centenary Early Career Award, the Paulo Foundation, the Finnish Foundation for Cardiovascular Research and the Jane and Aatos Erkko Foundation.
Patient consent for publication Not required.

Ethics approval The study followed the principles of the Declaration of Helsinki. The Ethics Committee of the Northern Ostrobothnia Hospital District approved the research.

Provenance and peer review Not commissioned; externally peer reviewed.

Open access This is an open access article distributed in accordance with the Creative Commons Attribution Non Commercial (CC BY-NC 4.0) license, which permits others to distribute, remix, adapt, build upon this work non-commercially, and license their derivative works on different terms, provided the original work is properly cited, appropriate credit is given, any changes made indicated, and the use is non-commercial. See: http://creativecommons.org/licenses/by-nc/4.0/.

\section{ORCID iDs}

Meri-Maija Ollila http://orcid.org/0000-0002-6794-0369

Elisabet Stener-Victorin http://orcid.org/0000-0002-3424-1502

\section{REFERENCES}

1 March WA, Moore VM, Willson KJ, et al. The prevalence of polycystic ovary syndrome in a community sample assessed under contrasting diagnostic criteria. Hum Reprod 2010;25:544-51.

2 Rotterdam ESHRE/ASRM-Sponsored PCOS Consensus Workshop Group. Revised 2003 consensus on diagnostic criteria and longterm health risks related to polycystic ovary syndrome. Fertil Steril 2004;81:19-25.

3 Franks S. Polycystic ovary syndrome. N Engl J Med Overseas Ed 1995;333:853-61.

4 Lambert GW, Straznicky NE, Lambert EA, et al. Sympathetic nervous activation in obesity and the metabolic syndrome-causes, consequences and therapeutic implications. Pharmacol Ther 2010;126:159-72.

5 Stuckey MI, Tulppo MP, Kiviniemi AM, et al. Heart rate variability and the metabolic syndrome: a systematic review of the literature. Diabetes Metab Res Rev 2014;30:784-93.

6 Kakoly NS, Moran LJ, Teede HJ, et al. Cardiometabolic risks in PCOS: a review of the current state of knowledge. Expert Rev Endocrinol Metab 2019;14:23-33.

7 Sgoifo A, Carnevali L, Alfonso MdelosAP, et al. Autonomic dysfunction and heart rate variability in depression. Stress 2015;18:343-52.

8 Paniccia M, Paniccia D, Thomas S, et al. Clinical and non-clinical depression and anxiety in young people: a scoping review on heart rate variability. Autonomic Neuroscience 2017;208:1-14.

9 Wulsin LR, Horn PS, Perry JL, et al. Autonomic imbalance as a predictor of metabolic risks, cardiovascular disease, diabetes, and mortality. J Clin Endocrinol Metab 2015;100:2443-8.

10 Saranya K, Pal GK, Habeebullah S, et al. Assessment of cardiovascular autonomic function in patients with polycystic ovary syndrome. J Obstet Gynaecol Res 2014;40:192-9.

11 Tekin G, Tekin A, Kılıçarslan EB, et al. Altered autonomic neural control of the cardiovascular system in patients with polycystic ovary syndrome. Int J Cardiol 2008;130:49-55.

12 Yildirir A, Aybar F, Kabakci G, et al. Heart rate variability in young women with polycystic ovary syndrome. Ann Noninv Electrocard 2006;11:306-12.

13 Lambert EA, Teede H, Sari Cl, et al. Sympathetic activation and endothelial dysfunction in polycystic ovary syndrome are not explained by either obesity or insulin resistance. Clin Endocrinol 2015;83:812-9.

14 Sverrisdóttir YB, Mogren T, Kataoka J, et al. Is polycystic ovary syndrome associated with high sympathetic nerve activity and size at birth? Am J Physiol Endocrinol Metab 2008;294:E576-81.

15 Heart rate variability: standards of measurement, physiological interpretation and clinical use. Task force of the European Society of cardiology and the North American Society of pacing and electrophysiology. Circulation 1996;93:1043-65.

16 Brown ZA, Louwers YV, Fong SL, et al. The phenotype of polycystic ovary syndrome ameliorates with aging. Fertil Steril 2011;96:1259-65.

17 Ollila M-ME, Piltonen T, Puukka K, et al. Weight gain and dyslipidemia in early adulthood associate with polycystic ovary syndrome: prospective cohort study. J Clin Endocrinol Metab 2016;101:739-47. 
18 Ollila M-ME, Kaikkonen K, Järvelin M-R, et al. Self-reported polycystic ovary syndrome is associated with hypertension: a northern Finland birth cohort 1966 study. J Clin Endocrinol Metab 2019;104:1221-31.

19 Alberti KGMM, Zimmet PZ, Definition ZPZ. Definition, diagnosis and classification of diabetes mellitus and its complications. Part 1 : diagnosis and classification of diabetes mellitus. provisional report of a who consultation. Diabet Med 1998;15:539-53.

20 Ollila MM, West S, Keinänen-Kiukaanniemi S, et al. Overweight and obese but not normal weight women with PCOS are at increased risk of type 2 diabetes mellitus - a prospective, population-based cohort study. Hum Reprod 2017;32:423-31.

21 Karjula S, Morin-Papunen L, Auvinen J, et al. Psychological distress is more prevalent in fertile age and premenopausal women with PCOS symptoms: 15-year follow-up. J Clin Endocrinol Metab 2017:102:1861-9.

22 Taponen S, Martikainen H, Järvelin M-R, et al. Hormonal profile of women with self-reported symptoms of oligomenorrhea and/or hirsutism: Northern Finland birth cohort 1966 study. J Clin Endocrinol Metab 2003;88:141-7.

23 Taponen Set al. Prevalence of polycystic ovaries in women with self-reported symptoms of oligomenorrhoea and/or hirsutism: Northern Finland birth cohort 1966 study. Human Reproduction 2004;19:1083-8.

24 Billman GE. The LF/HF ratio does not accurately measure cardiac sympatho-vagal balance. Front Physiol 2013;4:26.

25 Furlan R, Porta A, Costa F, et al. Oscillatory patterns in sympathetic neural discharge and cardiovascular variables during orthostatic stimulus. Circulation 2000;101:886-92

26 Pagani M, Somers V, Furlan R, et al. Changes in autonomic regulation induced by physical training in mild hypertension. Hypertension 1988;12:600-10.

27 Julien C. The enigma of Mayer waves: facts and models. Cardiovasc Res 2006;70:12-21.

28 Veijola J, Jokelainen J, Läksy K, et al. The Hopkins symptom Checklist-25 in screening DSM-III-R axis-I disorders. Nord $\mathrm{J}$ Psychiatry 2003;57:119-23.

29 Fauser BCJM, Tarlatzis BC, Rebar RW, et al. Consensus on women's health aspects of polycystic ovary syndrome (PCOS): the Amsterdam ESHRE/ASRM-Sponsored 3rd PCOS Consensus Workshop Group. Fertil Steril 2012;97:28-38.

30 Giallauria F, Palomba S, Manguso F, et al. Abnormal heart rate recovery after maximal cardiopulmonary exercise stress testing in young overweight women with polycystic ovary syndrome. Clin Endocrinol 2008;68:88-93.
31 Thayer JF, Yamamoto SS, Brosschot JF. The relationship of autonomic imbalance, heart rate variability and cardiovascular disease risk factors. Int J Cardiol 2010;141:122-31.

32 Hashim ZH, Hamdan FB, Al-Salihi AR. Autonomic dysfunction in women with polycystic ovary syndrome. Iran J Reprod Med 2015;13:27-34.

33 Vaz M, Jennings G, Turner A, et al. Regional sympathetic nervous activity and oxygen consumption in obese normotensive human subjects. Circulation 1997;96:3423-9.

34 Di Domenico K, Wiltgen D, Nickel FJ, et al. Cardiac autonomic modulation in polycystic ovary syndrome: does the phenotype matter? Fertil Steril 2013;99:286-92.

35 Shorakae S, Ranasinha S, Abell S, et al. Inter-related effects of insulin resistance, hyperandrogenism, sympathetic dysfunction and chronic inflammation in PCOS. Clin Endocrinol 2018;89:628-33.

36 Stauss HM. Identification of blood pressure control mechanisms by power spectral analysis. Clin Exp Pharmacol Physiol 2007;34:362-8.

37 Malliani A, Pagani M, Lombardi F, et al. Cardiovascular neural regulation explored in the frequency domain. Circulation 1991;84:482-92.

38 Kiviniemi AM, Frances MF, Tiinanen S, et al. $\alpha$-Adrenergic effects on low-frequency oscillations in blood pressure and $R-R$ intervals during sympathetic activation. Exp Physiol 2011;96:718-35.

39 Day F, Karaderi T, Jones MR, et al. Large-scale genome-wide meta-analysis of polycystic ovary syndrome suggests shared genetic architecture for different diagnosis criteria. PLoS Genet 2018;14:e1007813.

40 Teede HJ, Misso ML, Costello MF, et al. Recommendations from the International evidence-based guideline for the assessment and management of polycystic ovary syndrome. Clin Endocrinol 2018;89:251-68.

41 Thomson RL, Buckley JD, Noakes M, et al. Heart rate recovery improves after weight loss in overweight and obese women with polycystic ovary syndrome. Fertil Steril 2010;93:1173-8.

42 Giallauria F, Palomba S, Maresca L, et al. Exercise training improves autonomic function and inflammatory pattern in women with polycystic ovary syndrome (PCOS). Clin Endocrinol 2008;69:792-8.

43 Stener-Victorin E, Jedel E, Janson PO, et al. Low-Frequency electroacupuncture and physical exercise decrease high muscle sympathetic nerve activity in polycystic ovary syndrome. Am J Physiol Regul Integr Comp Physiol 2009;297:R387-95.

44 Maser RE, Lenhard MJ. An overview of the effect of weight loss on cardiovascular autonomic function. Curr Diabetes Rev 2007;3:204-11. 